\title{
Reply to the letter to the editor written by Dr. Philip Ng
}

\author{
Go Miyano - Toshiaki Takahashi · Satoko Shiyanagi • \\ Geoffrey J. Lane $\cdot$ Atsuyuki Yamataka
}

Accepted: 15 October 2012/ Published online: 24 November 2012

(c) Springer-Verlag Berlin Heidelberg 2012

Dear Dr. Philip Ng,

Thank you for your constructive comments about our manuscript, "Laparoscopy-assisted percutaneous endoscopic gastrostomy (Lap-PEG) using a Funada-kit 2 device".

We agree fully with him that the technique of laparoscopic gastrostomy insertion (Lap-G) is simple and widely used around the world. However, the advantages of our Lap-PEG procedure are that there is no need for blind fixation of the stomach to the abdominal wall and that the gastrostomy site can be chosen under both laparoscopic and endoscopic control. We also believe that our Lap-PEG can lower the risk for peritubal/intraperitoneal leakage because after the stomach wall is fixated with the Funada-kit 2 device, the PEG procedure is used for gastrostomy tube (G-Tube) insertion, where the G-Tube can be fixated tightly to the stomach and abdominal wall, without exteriorization of the stomach wall through the cannula port or enlargening the skin incision, both required during Lap-G. Although our series is only small, we have had no incidence of peritubal/ intraperitoneal leakage after our Lap-PEG.

Sincerely,

Go Miyano

G. Miyano $(\bowtie) \cdot$ T. Takahashi $\cdot$ S. Shiyanagi ·

G. J. Lane · A. Yamataka

Department of Pediatric General and Urogenital Surgery,

Juntendo University School of Medicine, 2-1-1 Hongo,

Bunkyo-ku, Tokyo 113-8421, Japan

e-mail: go1993@hotmail.co.jp 